\title{
Keramische Schwämme - Anwendungspotenzial monolithischer Netzstrukturen als katalytische Packungen
}

\author{
Andreas Reitzmann*, Florina Corina Patcas, \\ Bettina Kraushaar-Czarnetzki
}

\begin{abstract}
Keramische Schwämme, in der Literatur oft auch als Schäume oder „foams“ bezeichnet, bieten durch ihre hochporöse und allseitig fluiddurchlässige Struktur viel versprechende Voraussetzungen für den Einsatz als katalytische Packungen in Festbettreaktoren. Die hier zusammengefassten Ergebnisse aus der Fachliteratur zeigen, dass potenzielle Anwendungen insbesondere für Reaktionen bestehen, bei denen hohe Fluiddurchsätze gefordert sind, starke Wärmetönungen auftreten und/oder der Stofftransport die Kinetik beeinträchtigt. Durch keramische Schwammpackungen lassen sich offenbar die Vorzüge von Formkörperschüttungen und Wabenkörpern vereinen. Anders als bei diesen Festbetten gibt es bei Schwammpackungen noch große Defizite in der quantitativen Beschreibung von Fluiddynamik, Wärme- und Stofftransport. So soll dieser Beitrag auch den Forschungsbedarf aufzeigen, der notwendig ist, um belastbare quantitative Kriterien für einen zweckmäßigen Einsatz katalytischer Schwammpackungen ableiten zu können.
\end{abstract}

\section{Einleitung}

Schwämme lassen sich nicht nur aus Polymeren fertigen, sondern auch aus Metallen und aus diversen oxidischen und carbidischen Keramiken. Ein gemeinsames Merkmal aller Schwämme ist ihre hochporöse und allseitig fluiddurchlässige Netzstruktur. Im Mittelpunkt dieses Artikels sollen keramische Schwämme stehen, da hier Anforderungen an Temperaturstabilität und texturelle Eigenschaften besonders flexibel gestaltet werden können.

Keramische Schwämme wurden ursprünglich als Filter für Metallschmelzen entwickelt [1]. Inzwischen werden zunehmend auch Anwendungen in der Verfahrenstechnik untersucht, beispielsweise als Katalysatorträger, Porenbrenner, Partikelfilter, Wärmetauscher oder Knochenimplantate [2 - 6]. Bei genauer Betrachtung der verfahrenstechnischen Studien, insbesondere auch im Bereich der katalytischen Reaktionstechnik, stellt man fest, dass für individuelle Schwammproben spezielle Anwendungen demonstriert wurden. Auch wenn die Ergebnisse zum Teil sehr ermuti- gend sind und Vorteile gegenüber herkömmlichen Packungen oder Einbauten aufzeigen, so haben sie doch wenig Aussagekraft für andere Situationen. Der wichtigste Grund dafür sind Defizite im Verständnis und in der quantitativen Beschreibung von Transportvorgängen in festen Schwämmen, d.h. von Impuls-, Wärme- und Stofftransport.

\section{Grundlagen}

\subsection{Morphologie}

Feste Schwämme besitzen eine hochporöse, offenzellige Struktur bestehend aus verbundenen Stegen (s. Abb. 1). Stege und Hohlräume bilden zwei kontinuierliche Netzwerke, die sich gegenseitig durchdringen. In der Literatur werden die Begriffe "Schaum“ bzw. "foam“ verwendet. Jedoch bestehen Schäume definitionsgemäß aus geschlossenen, runden Blasen. In einem Schaum ist die Hohlraumphase also nicht kontinuierlich, sondern dispers. Der Begriff „Schwamm“ ist dagegen unmissverständlich und wird deswegen hier angewendet.
Ein gemeinsames Merkmal aller Schwämme ist ihre hochporöse und allseitig fluiddurchlässige Netzstruktur. 

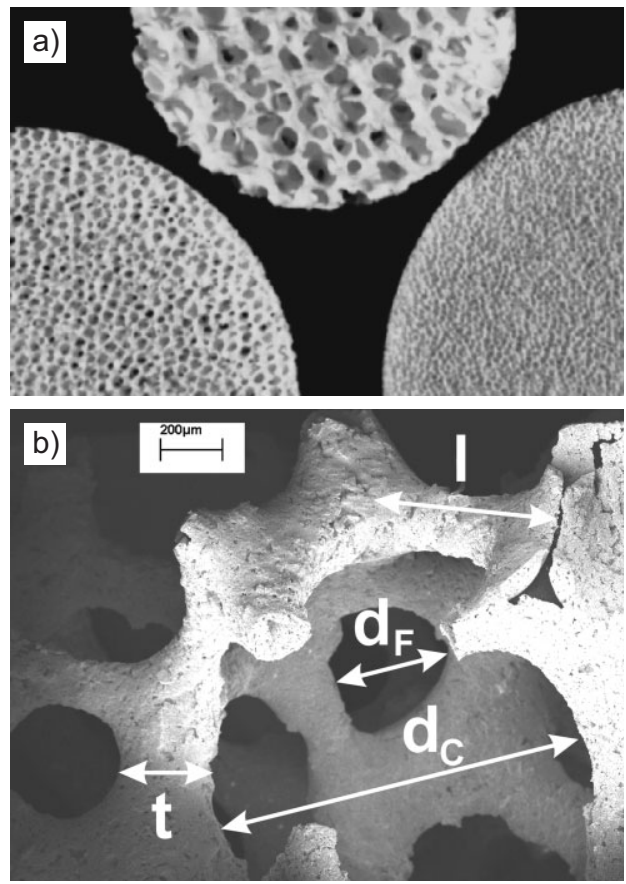

Abbildung 1. a) Keramische Schwämme mit 45 , 10 und 65 PPI (von links nach rechts); b) Detailaufnahme der Struktur mit geometrischen Größen (Stegdicke $t$, Steglänge $I$, Zellendurchmesser $d_{c}$ und Fensterdurchmesser $d_{F}$ ).

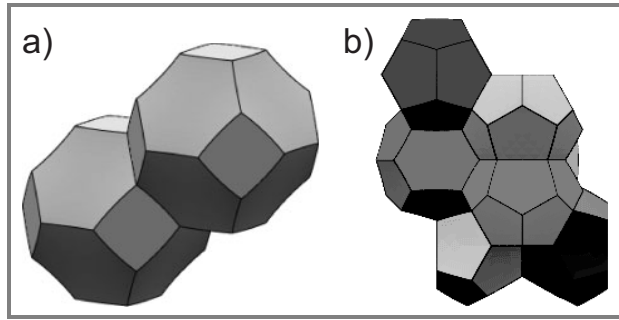

Abbildung 2. Kelvin-Packung aus gekappten Oktaeder (a) und Weaire-Phelan-Packung (b) [9].

Folgende Elemente können in der Schwammstruktur beobachtet werden:

Die unregelmäßige Schwammstruktur kann zu Modellierungszwecken durch bestimmte reguläre Netzwerke vereinfacht dargestellt werden.
- Stege, meist in Form von trigonalen Prismen, jeweils vier treffen sich in einem Knoten;

- Zellen, polyedrische Räume, jeweils drei Zellen grenzen an einen Steg;

- Fenster, polygonal, mit durchschnittlich fünf Seiten; jeweils zwei Zellen kommunizieren durch ein Fenster.

Die unregelmäßige Schwammstruktur kann $\mathrm{zu}$ Modellierungszwecken durch bestimmte reguläre Netzwerke vereinfacht dargestellt werden. Beispiele dafür sind eine Packung aus gekappten Oktaeder nach Kelvin [7] oder die Weaire-Phelan-Packung [8] (s. Abb. 2 [9]).

\subsection{Herstellung}

Prinzipiell gibt es zwei Möglichkeiten zur Herstellung der Schwämme: das Aufschäumen von Keramiksuspensionen und das Beschichten von Polymerschwämmen mit Keramiksuspensionen zum Erzeugen einer Replik. Mit der ersten Methode werden Schwämme mit einem großen Anteil an geschlossenen Zellen und relativ geringer Porosität erhalten. Die Replikmethode, wenn optimal durchgeführt, erlaubt die Erzeugung offenzelliger Schwämme. Nach der Beschichtung wird das Polymertemplat durch Verbrennen entfernt und anschließend der Keramikgrünkörper durch Hochtemperatursintern gefestigt. Wie man an Bruchund Schnittstellen erkennen kann, bleibt in den Stegen und Knoten eines keramischen Schwammes ein Hohlraum dort zurück, wo sich ursprünglich das Polymertemplat befand (s. Abb. 1b) $[10,11]$.

\subsection{Wichtige Stoffeigenschaften und geometrische Merkmale}

Die folgenden Eigenschaften können bei der Herstellung von keramischen Schwämmen nach der Replikmethode eingestellt werden:

- Das Material, d.h. die Element- und Phasenzusammensetzung, wird durch die $\mathrm{Zu}$ sammensetzung des Beschichtungsschlickers und die Sintertemperatur festgelegt. Durch die Auswahl des Materials kann man technische Eigenschaften wie Wärmeleitfähigkeit, maximale Betriebstemperatur, Härte und Thermoschockbeständigkeit einstellen $[10,11]$.

- Stegdicke, Zell- und Fenstergröße sind durch die Morphologie des Polymerschwammes vorgegeben, werden aber durch die Dicke der Keramikschicht und die Schwindung beim Trocknen und Sintern beeinflusst. Die so genannte „PPI“-Zahl (pores per inch) ist eine ungenaue Größe. Sie unterscheidet nicht zwischen Zellen und Fenstern. Traditionell bezieht sie sich nicht auf den keramischen Schwamm, sondern auf das Polymertemplat, aus dem er entstand. Gängige Werte bewegen sich zwischen 5 und 100 PPI (s. Abb. 1a).

- Der Leerraumanteil $\varepsilon$ ergibt sich aus Stegdicke, Zell- und Fenstergröße und hat bei Keramikschwämmen Werte zwischen 75 $90 \%$.

Da die Porenzahl PPI, wie erwähnt, nur eine grobe Abschätzung der Zellgröße erlaubt, sind für die Bestimmung der tatsächlichen Maße 
der Stege $(t, l)$, Fenster $\left(d_{\mathrm{F}}\right)$ und Zellen $\left(d_{\mathrm{C}}\right)$ mikroskopische Bilder unentbehrlich, die manuell oder rechnerisch ausgewertet werden müssen (s. Abb. 1b).

Die Gesamtporosität der Schwämme entspricht nicht nur dem Leerraumanteil $\varepsilon$. Zusätzlich müssen die Hohlräume in den Stegen und die Porosität der Keramik selbst berücksichtigt werden [10]. Für die Bestimmung des Leerraumanteils können Pyknometrie (mit einer nicht benetzenden Flüssigkeit) oder bildverarbeitende Methoden (mikroskopische Schliffbilder oder NMR-Tomographie) eingesetzt werden. Zwischen dem Leerraumanteil und der Dichte des Schwammes $(\rho)$ und der hohlen Stege $\left(\rho_{\mathrm{S}}\right)$ besteht die Beziehung:

$\varepsilon=1-\frac{\rho}{\rho_{S}}$

Die experimentelle Ermittlung der geometrischen Oberfläche, ein wichtiges Merkmal für den Einsatz als Katalysatorträger, ist nach dem jetzigen Stand der Technik bei keramischen Schwämmen nicht möglich. Die Messung der BET-Oberfläche ergibt die Gesamtfläche mitsamt jener der Makro- und Mesoporen in der Keramik sowie der Hohlräume in den Stegen und ist insofern nicht brauchbar. Eine Abschätzung der geometrischen Oberfläche bezogen auf das Bettvolumen ist jedoch möglich. Dafür gibt es einige Modelle in der Literatur, z. B.:

- das Modell des hydraulischen Durchmessers [12], wobei der Porendurchmesser jedoch nicht klar definiert ist (s. Abschnitt 3):

$S_{\mathrm{V}}=\frac{4 \varepsilon}{d_{\mathrm{p}}}$

- basierend auf der Kelvin-Packung [10]:

$S_{\mathrm{V}}=\frac{4,82}{d_{\mathrm{F}}+t} \sqrt{(1-\varepsilon)}$

\section{Technische Eigenschaften für den Einsatz als Reaktorpackung}

Untersuchungen über technisch relevante Eigenschaften von Schwämmen sind in der Literatur sehr ungleichmäßig gewichtet. Während mechanische Eigenschaften intensiv untersucht und quantitativ bestimmt wurden [7, 13], ist die Beschreibung von reaktionstechnisch relevanten Eigenschaften unzureichend. Ein immer wiederkehrendes Problem bleibt die Definition eines charakteristischen Merkmals oder Längenmaßes. Zunehmend wird der mikroskopisch gemessene so genannte Porendurchmesser $d_{\mathrm{p}}$ eingesetzt, wobei aber meistens unklar bleibt, ob damit die Fenster, die
Zellen oder ein Mittelwert aus beiden gemeint ist. Die geometrische Größen, die in Abschnitt 2.3 und $\mathrm{Abb}$. $1 \mathrm{~b}$ definiert wurden, wären besser geeignet, werden aber nur selten verwendet (z. B. Stegdicke t). Diese Problematik wird in den folgenden Betrachtungen über Korrelationen und Modelle für Druckverlust, Stoffund Wärmetransport in Schwammpackungen deutlich.

\subsection{Druckverlust}

Es gibt viele Untersuchungen über den Druckverlust in Schwämmen, da dieser eine relativ einfach zugängliche Größe darstellt. Als Basis für die Auswertung der Druckverlust-Geschwindigkeitsdaten wird die ForchheimerGleichung verwendet [14]:

$-\frac{\mathrm{d} P}{\mathrm{~d} x}=\frac{\eta}{k_{1}} w_{0}+\frac{\rho_{\mathrm{F}}}{k_{2}} w_{0}^{2}$

Die Permeabilitätskonstanten $k_{1}$ und $k_{2}$ sind von den Strukturmerkmalen des Schwammes abhängig. Sie stehen für den viskosen und Trägheitsanteil des Druckverlustbeiwerts.

Allgemein werden in Veröffentlichungen und Produktdatenblättern Werte der Permeabilitätskonstanten für einzelne Schwämme oder Schwammgruppen auf PPI-Angaben des Herstellers bezogen. Als charakteristische Länge dient nahezu ausschließlich der Porendurchmesser. Selten wurde versucht, diese Konstanten mit klar definierten Strukturmerkmalen zu korrelieren. Der jetzige Stand des Wissens in dieser Hinsicht ist in Tab. 1 zusammengefasst.

Ein Vergleich der Korrelationen mit Druckverlustmessungen ist in Abb. 3 exemplarisch für einen Schwamm mit 30 PPI und $\varepsilon=0,87$ dargestellt. Die Messdaten liegen zwischen den Kurven der empirischen Modelle [12, 15] und der Kurve, die durch Simulation der Strö-

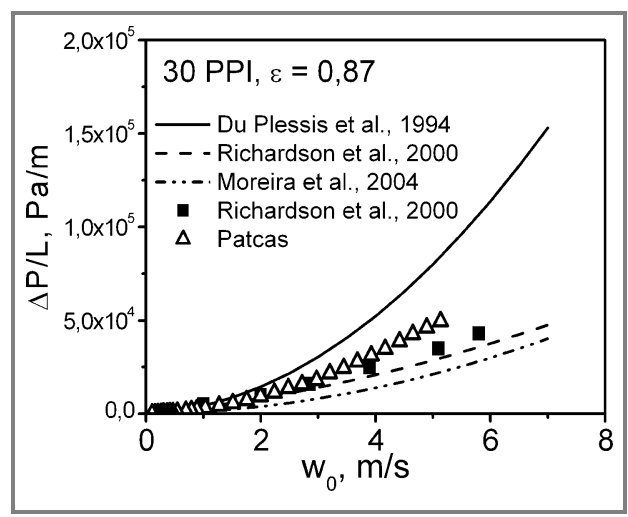

Abbildung 3. Vergleich von Messdaten (Symbole) mit berechneten Werte aus Literaturkorrelationen (Linien) $[12,15,16]$. 


\begin{tabular}{|c|c|c|c|c|}
\hline Modell & Korrelationen & Schwammeigenschaften & Bedingungen & Lit. \\
\hline $\begin{array}{l}\text { Kubische Zellen } \\
\text { (Navier-Stokes) }\end{array}$ & $\begin{array}{c}k_{1}=\frac{\varepsilon^{2} d_{\mathrm{p}}^{2}}{36 \chi(\chi-1)} ; \quad k_{2}=\frac{\varepsilon^{2}(3-\chi) d_{\mathrm{p}}}{2,05 \chi(\chi-1)} \\
\frac{1}{\chi}=\frac{3}{4 \varepsilon}+\frac{\sqrt{9-8 \varepsilon}}{2 \varepsilon} \cos \left\{\frac{4 \pi}{3}+\frac{1}{3} \cos ^{-1}\left[\frac{8 \varepsilon^{2}-36 \varepsilon+27}{(9-8 \varepsilon)^{3 / 2}}\right]\right\}\end{array}$ & $\begin{array}{l}\text { Metall } \\
45,60,100 \text { PPI } \\
\varepsilon=0,97\end{array}$ & $w_{0}=0-0,8 \mathrm{~m} / \mathrm{s}$ & [16] \\
\hline Ergun (empirisch) & $\begin{array}{c}k_{1}=\frac{\varepsilon^{3}}{a\left(\frac{S_{\mathrm{v}}}{1-\varepsilon}\right)^{2}(1-\varepsilon)^{2}} ; \quad k_{2}=\frac{\varepsilon^{3}}{\beta\left(\frac{S_{\mathrm{v}}}{1-\varepsilon}\right)(1-\varepsilon)} \\
a=9,7310^{2} d_{\mathrm{p}}^{0,743}(1-\varepsilon)^{-0,0982} \\
\beta=3,6810^{-4} d_{\mathrm{p}}^{-0,7523}(1-\varepsilon)^{0,07158}\end{array}$ & $\begin{array}{c}a-\mathrm{Al}_{2} \mathrm{O}_{3} \\
10,30,45,65 \text { PPI } \\
\varepsilon=0,82\end{array}$ & $w_{0}=1-8 \mathrm{~m} / \mathrm{s}$ & [12] \\
\hline Ergun (empirisch) & $k_{1}=\frac{\varepsilon^{3} d_{\mathrm{p}}^{0,264}}{1,36 \cdot 10^{8}(1-\varepsilon)^{2}} ; \quad k_{2}=\frac{\varepsilon^{3} d_{\mathrm{p}}^{-0,24}}{1,8 \cdot 10^{4}(1-\varepsilon)}$ & $\begin{array}{c}\mathrm{SiC}_{-\mathrm{Al}_{2} \mathrm{O}_{3}} \\
8 \mathrm{PPI}, \varepsilon=0,94 \\
20 \mathrm{PPI}, \varepsilon=0,88 \\
45 \mathrm{PPI}, \varepsilon=0,76\end{array}$ & $w_{0}=0-20 \mathrm{~m} / \mathrm{s}$ & [15] \\
\hline
\end{tabular}

Tabelle 1. Korrelationen zur Berechnung des Druckverlustes in Schwammpackungen.

mung in einem kubischen Steggitter mit Hilfe der Navier-Stokes-Gleichungen [16] erhalten wurde. Dieses Beispiel deutet an, dass der Einfluss von strukturellen und texturellen Eigenschaften der Schwämme auf den Druckverlust mit den bestehenden Korrelationen noch nicht umfassend wiedergegeben werden kann.

\subsection{Stoffübergang Fluid/Feststoff}

Zur Stoffübertragung zwischen Schwammoberfläche und Fluid sind kaum Literaturdaten vorhanden. Die einzigen beiden Korrelationen für die Ermittlung von Stoffübergangskoeffizienten sind in Tab. 2 aufgeführt. In beiden Fällen wurde die Oxidation von Kohlenmonoxid an mit $\mathrm{Pt} / \mathrm{Al}_{2} \mathrm{O}_{3}$ oder $\mathrm{Pd} / \mathrm{Al}_{2} \mathrm{O}_{3}$ beschichteten Schwämmen im stoffübergangskontrollierten Bereich angewandt $[17,18]$. Die Ergebnisse der Korrelationen der beiden Arbeitsgruppen unterscheiden sich jedoch um einen Faktor von 1,5 bis 2 .

\subsection{Wärmetransport}

Zum Wärmetransport tragen bekanntlich Leitung, erzwungene und freie Konvektion sowie Strahlung bei. Experimentelle Untersuchungen an Schwämmen beschränken sich bisher auf einzelne Teilaspekte (z. B. Ruhewärmeleitfähigkeiten, Konvektion zwischen Schwammoberfläche und Fluid) oder die Bestimmung von effektiven Wärmeübertragungsgrößen, die verschiedene Mechanismen einschließen. Zudem wurden nur einzelne Schwämme mit speziellen Eigenschaften betrachtet. So zeigt Tab. 3 beispielhafte Korrelationen für Schwammpackungen, die aber nur für die angegebenen Materialien und Bedingungen gelten [19 - 25]. Eine aktuelle Übersicht über weitere Modelle und Korrelationen findet sich in [26].

\subsection{Vergleiche zwischen Schwämmen, Schüttungen und Wabenkörpern}

Vergleichende Untersuchungen zwischen Schwämmen und anderen Packungen sind

\begin{tabular}{|c|c|c|c|c|}
\hline Ausgangsmodell & Korrelationen & Schwammeigenschaften & Bedingungen & Lit. \\
\hline $\begin{array}{l}\text { Partikelpackung } \\
\text { (empirisch) }\end{array}$ & $\begin{array}{c}\varepsilon j_{\mathrm{D}}=0,233 \mathrm{Re}_{\mathrm{S}}^{-0,416} ; \quad j_{\mathrm{D}}=\frac{k_{m} \mathrm{Sc}^{2 / 3}}{w_{0}} ; \\
\text { charakter. Länge: } 1 / S_{\mathrm{V}}\end{array}$ & $\begin{array}{l}a-\mathrm{Al}_{2} \mathrm{O}_{3} \\
30 \mathrm{PPI} \\
\varepsilon=0,82\end{array}$ & $w_{0}=1-8 \mathrm{~m} / \mathrm{s}$ & [17] \\
\hline $\begin{array}{l}\text { Kubische Zellen } \\
\text { (empirisch) }\end{array}$ & $\begin{array}{l}\quad \frac{k_{\mathrm{m}} t}{D_{\mathrm{m}}}=1,1 \mathrm{Re}_{\mathrm{t}}^{0,43} \mathrm{Sc}^{1 / 3} \\
\text { charakter. Länge: Stegstärke } t\end{array}$ & $\begin{array}{l}\text { Fe-Cr-Leg. } \\
\text { 10, 20, 40 PPI } \\
\varepsilon=0,95\end{array}$ & $w_{0}=0,8-2,6$ & {$[18]$} \\
\hline
\end{tabular}

Tabelle 2. Korrelationen zur Berechnung des Stoffübergangskoeffizienten in Schwammpackungen. 


\begin{tabular}{|c|c|c|c|c|}
\hline $\begin{array}{l}\text { Art der Wärme- } \\
\text { übertragung }\end{array}$ & Korrelationen & $\begin{array}{l}\text { Schwamm- } \\
\text { eigenschaften }\end{array}$ & Bedingungen & Lit. \\
\hline \multirow[t]{3}{*}{$\begin{array}{l}\text { Konvektion: } \\
\text { Schwamm - Fluid }\end{array}$} & $\begin{array}{c}\frac{h d_{\mathrm{h}}}{\lambda_{\mathrm{f}}}=c\left(\frac{\operatorname{Re}_{\mathrm{dh}}}{\varepsilon}\right)^{\mathrm{m}} \operatorname{Pr}^{1 / 3} \\
m=0,27-0,47, c=0,8-1,6 \text { (abhängig von PPI) } \\
\text { charakter. Länge: hydraul. Durchmesser } d_{\mathrm{h}}=\frac{4 \varepsilon}{S_{\mathrm{V}}}\end{array}$ & $\begin{array}{l}\text { Cordierit, } \\
10,20,30,50 \text { PPI } \\
\varepsilon=0,85\end{array}$ & $\begin{array}{l}\text { je nach PPI: } \\
\begin{aligned} 28 & <\operatorname{Re}<2840 \\
& \approx 300{ }^{\circ} \mathrm{C}\end{aligned}\end{array}$ & [19] \\
\hline & $\begin{array}{c}\qquad \frac{h t}{\lambda_{\mathrm{f}}}=1,2 \operatorname{Re}_{\mathrm{t}}^{0,43} \operatorname{Pr}^{1 / 3} \\
\text { charakter. Länge: Stegdicke } t \\
\text { Modell: prismatische Einheitszelle }\end{array}$ & $\begin{array}{c}\mathrm{Ni} / \mathrm{Cr}, \mathrm{Cu} \\
10,20 \mathrm{PPI} \\
\varepsilon=0,91-0,93\end{array}$ & $\begin{aligned} 20 & <\mathrm{Re}<240 \\
& \approx 300^{\circ} \mathrm{C}\end{aligned}$ & [20] \\
\hline & $\begin{array}{c}\frac{h S_{\mathrm{V}} d_{\mathrm{p}}^{2}}{\lambda_{\mathrm{f}}}=0,819\left[1-7,33\left(d_{\mathrm{p}} / L\right)\right] \operatorname{Re}^{0,36\left[1+15,5\left(d_{\mathrm{p}} / L\right)\right]} \\
\text { charakter. Länge: Porendurchmesser } d_{\mathrm{p}} \\
\text { Länge der Schwammprobe: } L\end{array}$ & $\begin{array}{c}92 \% \mathrm{Al}_{2} \mathrm{O}_{3} \\
10 \mathrm{PPI}, \varepsilon=0,87 \\
20 \mathrm{PPI}, \varepsilon=0,85 \\
30 \mathrm{PPI}, \varepsilon=0,84 \\
45 \mathrm{PPI}, \varepsilon=0,834\end{array}$ & $\begin{array}{c}5,1<\operatorname{Re}<564 \\
0,005<d_{\mathrm{p}} / L< \\
0,136 \\
\approx 70^{\circ} \mathrm{C}\end{array}$ & {$[21]$} \\
\hline \multirow[t]{2}{*}{$\begin{array}{l}\text { Ruhewärmeleit- } \\
\text { fähigkeit }\end{array}$} & $\begin{array}{c}\frac{\lambda_{0}}{\lambda_{\mathrm{f}}}=\left[\frac{2 t}{\left(1-\varepsilon_{\mathrm{s} 1}\right)\left(\lambda_{\mathrm{s}} / \lambda_{\mathrm{f}}\right)+\varepsilon_{\mathrm{s} 1}}+\frac{(1-2 t)}{\left(1-\varepsilon_{\mathrm{s} 2}\right)\left(\lambda_{\mathrm{s}} / \lambda_{\mathrm{f}}\right)+\varepsilon_{\mathrm{s} 2}}\right]^{-1} \\
\varepsilon_{\mathrm{s} 1}=(1-2 t)^{2} ; \varepsilon_{\mathrm{s} 2}=(1-4 t)^{2} \\
\text { charakter. Länge: Stegdicke } t \\
\text { kubisches Strukturmodell mit zwei Porositäten }\end{array}$ & $\begin{array}{c}\text { Stabilisiertes } \mathrm{ZrO}_{2} \\
\varepsilon=0,85-0,87\end{array}$ & $27-527^{\circ} \mathrm{C}$ & {$[22]$} \\
\hline & $\lambda_{0}=0,35\left(\varepsilon \lambda_{\mathrm{f}}+(1-\varepsilon) \lambda_{\mathrm{s}}\right)+\frac{0,75}{\left(\frac{\varepsilon}{\lambda_{\mathrm{f}}}+\frac{1-\varepsilon}{\lambda_{\mathrm{s}}}\right)}$ & $\begin{array}{c}\text { Al, Carbon } \\
5,10,20,40 \text { PPI; } \\
\varepsilon=0,91-0,97\end{array}$ & $\begin{array}{c}\text { Luft, Wasser } \\
25^{\circ} \mathrm{C}\end{array}$ & [23] \\
\hline \multirow[t]{2}{*}{$\begin{array}{l}1 \text { dim., Wärme- } \\
\text { durchgang }\end{array}$} & $\begin{array}{c}\frac{h_{\mathrm{eff}} d_{R}}{\lambda_{\mathrm{f}}}=2,49 \cdot 10^{-8} \varepsilon T^{3}+12,6 \operatorname{Re}_{\mathrm{s}}(1-\varepsilon) \\
\text { charakter. Länge: } 1 / \mathrm{S}_{\mathrm{V}}\end{array}$ & $\begin{array}{c}a-\mathrm{Al}_{2} \mathrm{O}_{3} / \mathrm{Mullit} \\
30 \mathrm{PPI} \\
\varepsilon=0,82\end{array}$ & $\begin{array}{c}d_{\mathrm{R}}=12,4 \mathrm{~mm} \\
700-900{ }^{\circ} \mathrm{C}\end{array}$ & [17] \\
\hline & $\begin{array}{c}\frac{h_{\text {eff }}}{\lambda_{\mathrm{f}} S_{\mathrm{V}}}=8,14 \cdot 10^{-11} \varepsilon T^{3}+0,0708 \operatorname{Re}_{\mathrm{s}}(1-\varepsilon) \\
\text { charakter. Länge: } 1 / \mathrm{S}_{\mathrm{V}}\end{array}$ & $\begin{array}{l}a-\mathrm{Al}_{2} \mathrm{O}_{3} / \text { Mullit } \\
\text { mit } \gamma-\mathrm{Al}_{2} \mathrm{O}_{3} \text { washcoat } \\
30 \mathrm{PPI}, \varepsilon=0,874\end{array}$ & $\begin{array}{c}0,2<\operatorname{Re}_{\mathrm{s}}<1,7 \\
25-400^{\circ} \mathrm{C}\end{array}$ & {$[24]$} \\
\hline \multirow[t]{2}{*}{$\begin{array}{l}2 \text { dim., effektive } \\
\text { Wärmeleitfähigkeit }\end{array}$} & $\begin{array}{c}\lambda_{\text {eff,radial }}=6,84 \cdot 10^{-5} 1 / S_{\mathrm{V}} T^{3}+42,2 \operatorname{Re}_{\mathrm{s}}(1-\varepsilon) \lambda_{\mathrm{f}} \\
\text { charakter. Länge: } 1 / \mathrm{S}_{\mathrm{V}}\end{array}$ & $\begin{array}{l}a-\mathrm{Al}_{2} \mathrm{O}_{3} / \text { Mullit } \\
30 \text { PPI, } \varepsilon=0,87\end{array}$ & $\begin{array}{c}2,86<\mathrm{Re}_{\mathrm{s}}<14,4 \\
25-400^{\circ} \mathrm{C}\end{array}$ & [24] \\
\hline & $\begin{array}{c}\frac{\lambda_{\text {eff }, \mathrm{i}}}{\lambda_{\mathrm{f}}}=\frac{\lambda_{0}}{\lambda_{\mathrm{f}}}+\frac{\mathrm{Pe}}{K_{\mathrm{i}}}, \mathrm{i}=\text { radial, axial } \\
K_{\text {radial }}=0,54(\mathrm{Ni}-\mathrm{Cr})-6,96 \text { (Tongeb. SiC) } \\
K_{\text {axial }}=0,037 \text { (Ni-Cr) }-18,52 \text { (gesintertes } \mathrm{SiC} \text { ) } \\
\text { charakter. Länge: Porendurchmesser } d_{\mathrm{p}}\end{array}$ & $\begin{array}{c}\text { Ni-Cr, } \\
10 \mathrm{PPI}, \varepsilon=0,9 \\
\text { gesintertes } \mathrm{SiC} \text {, Tongeb. } \\
\mathrm{SiC}, \text { Cordierit } \\
10,20,45 \mathrm{PPI} \\
\varepsilon=0,76,0,81\end{array}$ & $\begin{array}{c}\mathrm{u} \leq 1 \mathrm{~m} / \mathrm{s} \\
50-500^{\circ} \mathrm{C}\end{array}$ & [25] \\
\hline
\end{tabular}

Tabelle 3. Korrelationen zur Berechnung verschiedener Wärmetransportkoeffizienten in Schwammpackungen.

selten in der Literatur zu finden. So lassen sich derzeit die Eigenschaften katalytischer Schwammpackungen nur qualitativ im Vergleich zu anderen Katalysatorstrukturen, z. B. Formkörperschüttungen oder Wabenkörpern, einordnen und bewerten.

Folgende Erkenntnisse lassen sich aus den bisher vorhandenen Untersuchungen ziehen:

- Wie Schüttschichten sind feste Schwämme in allen Raumrichtungen gleichermaßen fluiddurchlässig. Ungleichverteilungen der Durchströmung werden bei ausreichender Strömungslänge ausgeglichen und setzen sich nicht wie bei Wabenkörpern bis an der Austritt fort.
- Wegen ihres hohen Hohlraumanteils weisen feste Schwämme bei Durchströmung einen geringeren Druckverlust als Partikelschüttungen, aber einen höheren als Wabenkörper auf, wenn man den Vergleich bei gleicher Oberfläche bezogen auf das Packungsvolumen zieht (s. Abb. 4).

- Anders als bei Wabenkörpern sind in festen Schwämmen Strömungsablösung und Wirbelbildung realisierbar. Stoff- und Wärmeübertragung zwischen Fluid und Schwammoberfläche können dadurch verbessert werden. Tatsächlich werden im Vergleich zu geometrisch ähnlichen Wabenkörpern aus gleichem Material höhere Stoffübergangs- 


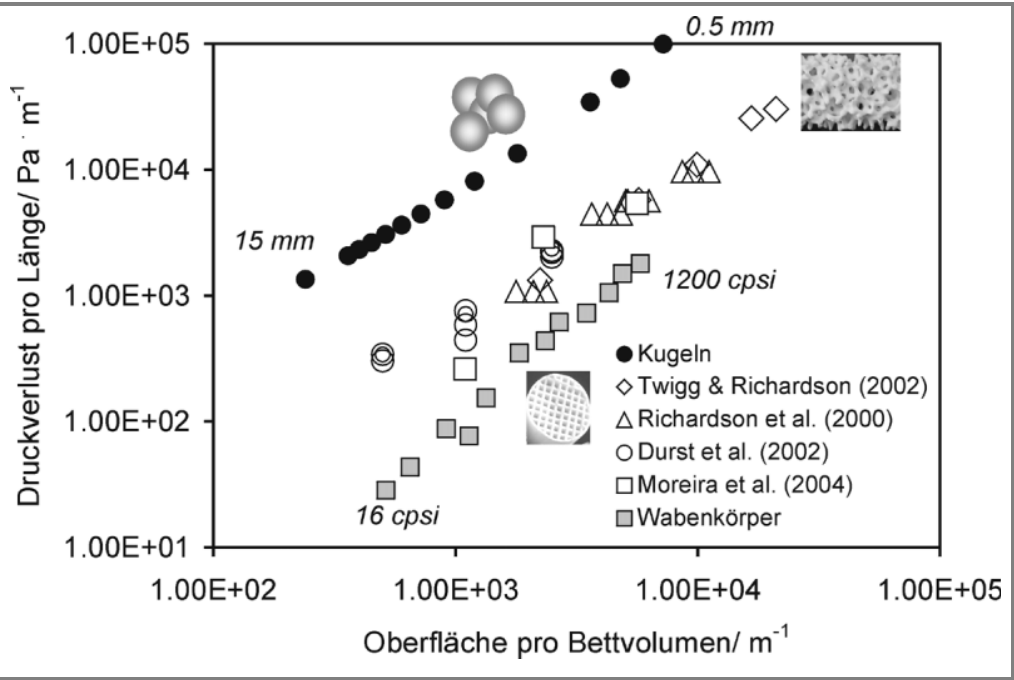

Abbildung 4. Druckverlust von Kugelschüttungen (berechnet nach Ergun, $\varepsilon=0,4$ ), Wabenkörpern (Daten aus [27], quadratische Kanäle, berechnet nach Darcy-Weißbach) und keramischen Schwämmen (aus $[6,12,15,25]$ ) als Funktion der spezifischen Oberflache $\left(w_{0}=1 \mathrm{~m} / \mathrm{s}\right)$.
Es ist zu erwarten, dass beim Einsatz von Schwämmen die günstigen Eigenschaften von Schüttschichten mit jenen von Wabenkörper vorteilhaft kombiniert werden können.
[18] und höhere konvektive Wärmeübergangskoeffizienten bestimmt [19].

- Die tortuose Struktur und der hohe Leerraumanteil führen zu einer radialen Vermischung über den gesamten Querschnitt von Schwammpackungen.

- Kennzahlen, die Stoffübergang und Druckverlust ins Verhältnis setzen, sind für Schwämme und Wabenkörper in der gleichen Größenordnung, aber deutlich größer als für Formkörperschüttungen [18].

- Im Vergleich zu Formköperschüttungen verstärkt bei Temperaturen $<400{ }^{\circ} \mathrm{C}$ die Wärmeleitung in der durchgehenden Feststoffphase [22], bei Temperaturen $>600{ }^{\circ} \mathrm{C}$ die Wärmestrahlung in der hochporösen Struktur [17] den Wärmetransport.

Es ist zu erwarten, dass beim Einsatz von Schwämmen die günstigen Eigenschaften von Schüttschichten mit jenen von Wabenkörper vorteilhaft kombiniert werden können. Für genauere Aussagen sind noch detaillierte Studien notwendig. Insbesondere muss ein gemeinsames Merkmal identifiziert werden, das einen aussagekräftigen Vergleich von Festbetten mit solch unterschiedlichen Topologien überhaupt ermöglicht. Ein sinnvolles Merkmal könnte die auf das Bettvolumen bezogene geometrische Oberfläche sein (s. Abschnitt 2.3). Diese Größe ist relevant, weil sie ein Maß für die Menge an Aktivphase des Katalysators ist, die pro Volumeneinheit in die Packung durch Beschichtung eingebracht werden kann, und weil sie anderseits den maximal erreichbaren Umsatz bei stoffübergangskontrollierten Prozessen vorgibt.

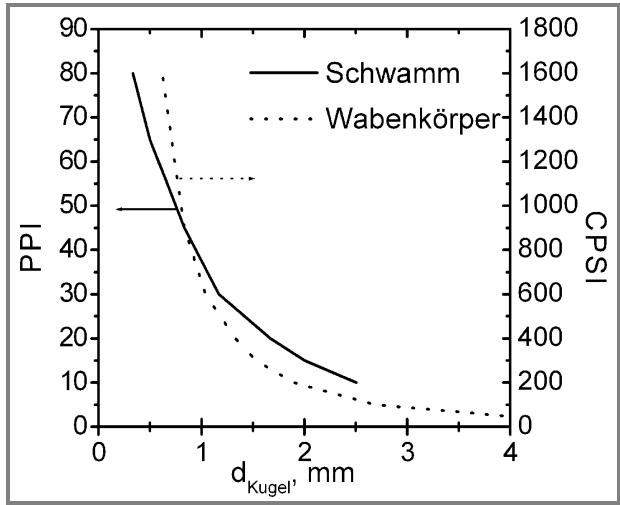

Abbildung 5. Korrespondenzdiagramm von Schwämmen ( $\varepsilon=0,8$, Daten [1]), Wabenkörpern (Daten aus [27]) und Kugelschüttungen $(\varepsilon=0,4)$ bei gleicher bettvolumenbezogener Oberfläche.

Abb. 5 veranschaulicht beispielhaft, wie Schwämme und Wabenkörper eine Schüttung aus Kugeln repräsentieren können, wenn die gleiche spezifische Oberfläche pro Packungsvolumen vorliegt. Im Fall der Schwämme wurde die Oberfläche nach Gl. (3) mit Daten der Fa. Hi-Tech-Ceramics [1] für einen Leerraumanteil von $\varepsilon=0,8$ berechnet, für die Kugelschüttung wurde eine Porosität $\varepsilon=0,4$ und für Wabenkörper Werte aus [27] verwendet.

\section{Feste Schwämme als Katalysatorträger}

Wegen der hohen Sintertemperaturen, die für die Gewährleistung einer ausreichenden mechanischen Festigkeit benötigt werden, können die Schwämme meistens nicht direkt aus den katalytisch aktiven Komponenten hergestellt werden. Feste Schwämme werden daher in der Regel als Träger eingesetzt und müssen mit dem Katalysator beschichtet werden.

Die Aufbringung einer gleichmäßigen, kontinuierlichen Schicht auf das Stegnetzwerk stellt keine triviale Aufgabe dar. Vor allem die Beschichtung von Schwämmen mit glatter Stegoberfläche ist besonders aufwändig. Bestimmte Vorbereitungsschritte wie die Oxidation bei höheren Temperaturen (bei Schwämmen aus FeCr-Legierung oder $\mathrm{SiC}$ ) oder das Anätzen mit Säuren können die Haftung der katalytisch aktiven Schichten verbessern [28].

In den meisten Fällen werden Tauchverfahren („Dip-Coating“) mit Partikelsuspensionen angewendet [28 - 32]. Der aufzubringende fein gemahlene Feststoff wird mit einer Flüssigkeit und eventuell mit Zusätzen (Peptisatoren, Verdickungs- und Bindemittel) zu einer stabilen Suspension vermischt. Alternativ kann ein Sol eingesetzt werden $[28,33]$. Die Viskosität von 
Suspension oder Sol muss an die Porengröße des Schwammes angepasst werden: Das Fluid muss ausreichend flüssig sein, um den Schwamm zu penetrieren und um den Überschuss abtropfen zu lassen, gleichzeitig zäh genug, damit eine Schicht haften kann. Die Schichtdicke kann z. B. über die Feststoffkonzentration [33], die Viskosität [28] oder die Anzahl der Beschichtungsvorgänge [30, 33] eingestellt werden. Die Viskosität kann durch Zugabe von Peptisatoren und/oder organischen Zusatzstoffen in bestimmten Grenzen unabhängig vom Feststoffanteil variiert werden. Ein Vergleich in [28] legt nahe, dass durch Sol-Gel-Methoden eine homogenere $\gamma-\mathrm{Al}_{2} \mathrm{O}_{3}$ Schicht auf Metallschwämmen erzeugt werden kann als durch Einsatz von Suspensionen.

In einigen Fällen wurden Schwämme auch durch Eintauchen in homogene Lösungen der Vorläufersalze mit Katalysatoren, insbesondere Edelmetallen, beschichtet [34 - 44]. Bei dieser Methode kann man allerdings keine hohe Dispersion und stabile Haftung erzielen.

Neben den Tauchverfahren existiert bei den Schwämmen die Möglichkeit, Katalysatorpartikel aus strömenden Suspensionen mit Hilfe der Schwämme herauszufiltern [28, 31, 32]. Im Fall von $\mathrm{CsVO}_{3} \cdot \mathrm{MoO}_{3}$ - und $\mathrm{Cs}_{2}\left(\mathrm{SO}_{4}\right)_{2}$. $\mathrm{V}_{2} \mathrm{O}_{5}$-Katalysatoren wurde mit dieser Methode im Gegensatz zu Tauchverfahren eine gleichmäßige Katalysatorschicht erzeugt, ohne dass die überschüssige Flüssigkeit aufwändig aus dem Porensystem entfernt werden musste [31].

Speziell Zeolithschichten lassen sich auch durch trägergestützte Kristallisation aufbringen. Der Schwamm dient dabei nicht nur als Träger, sondern ist gleichzeitig die Siliciumoder Aluminiumquelle für das Wachstum der Zeolithkristalle. Bei dieser Methode lässt sich ein besonders enger Verbund zwischen Zeolithkristallen und Schwammoberfläche erzielen $[45,46]$.

Einen neuen Ansatz stellt die Beschichtung mit Katalysatorpartikeln durch Abscheidung aus einer Gas/Feststoff-Strömung dar [47, 48]. In [48] wird ein Verfahren vorgestellt, bei dem Katalysatorsynthese und Schwammbeschichtung in einem einzigen Apparat und Arbeitsschritt erfolgen. Der Katalysator besteht hier aus $\mathrm{V}_{2} \mathrm{O}_{5} / \mathrm{TiO}_{2}$-Nanopartikeln, die durch Flammensprühpyrolyse erzeugt und oberhalb der Flamme in einer Schwammpackung abgeschieden werden. Eine Ablagerung in einer Vorzugsrichtung zur Strömung kann unter bestimmten Bedingungen vermieden werden, da die Diffusion wesentlich zum Partikeltransport beiträgt. Schichtdicke sowie Größe und $\mathrm{Zu}$ sammensetzung der Partikel können durch geeignete Wahl der Betriebsbedingungen flexibel gestaltet werden.

\section{Katalytische Anwendungen}

Als Katalysatoren bzw. Katalysatorträger können Schwämme insbesondere dann Vorteile gegenüber herkömmlichen Festbetten bieten, wenn hohe Fluiddurchsätze gefordert sind, der Transport der Reaktanden aus der Gasphase zur Katalysatoroberfläche die Reaktionskinetik hemmt und/oder Reaktionen eine starke Wärmetönung aufweisen. Nach Kenntnis der Autoren gibt es noch keine technische Anwendung von katalytischen Schwämmen, aber die Zahl der Veröffentlichungen und Patente auf diesem Gebiet nimmt seit ein paar Jahren stark zu. Einige interessante Beiträge werden in den folgenden Abschnitten zusammengefasst.

\subsection{Katalytische Verbrennung}

Die katalytische Verbrennung zählt zu den ersten Anwendungen von Schwammpackungen als Katalysatoren [49 - 54]. Die untersuchten Brennstoffe sind Wasserstoff [49], aber vor allem Kohlenwasserstoffe wie Methan [52, 53], $\mathrm{C}_{2}$ - $\mathrm{C}_{4}$-Alkane [50 - 52] und Erdgas [54]. Die Schwämme bestehen in der Regel aus Mullit oder (stabilisiertem) $a-\mathrm{Al}_{2} \mathrm{O}_{3}$, und als katalytisch aktive Komponente wird fast immer Platin verwendet.

An katalytischen Schwämmen ist in einem weiten Luftzahlbereich eine stabile und schadstoffarme Verbrennung möglich. Bei den für die atmosphärische katalytische Verbrennung typischen Temperaturen von $800-1100{ }^{\circ} \mathrm{C}$ wird eine Vergiftung des Katalysators durch Chlor- und Schwefelverbindungen unterbunden. Die kurzen Kontaktzeiten von $\sim 10 \mathrm{~ms}$ erlauben eine sehr kompakte Bauweise der Brennkammern [52]. Ein Vergleich von Schwämmen ohne katalytische Beschichtung, den so genannten Porenbrennern [5], und solchen, die mit $\mathrm{LaMnO}_{3}$-Perowskit beschichtet waren, ergab bei der Verbrennung von Methan, dass insbesondere bei niedrigen Luftüberschüssen und geringen Leistungsdichten $\left(<190 \mathrm{~kW} / \mathrm{m}^{2}\right)$ die katalytische Verbrennung stabiler verläuft und die CO-Emissionen deutlich verringert werden können [53].

Die Wirkung von Schwammpackungen mit und ohne Platinbeschichtung wurde auch für hohe Einsatztemperaturen, wie sie für Gasturbinen typisch sind (mindestens $1200^{\circ} \mathrm{C}$ ) untersucht und verglichen. Es konnte nachgewiesen werden, dass bei katalytischer Verbrennung die $\mathrm{NO}_{\mathrm{x}}$-Emissionen deutlich verringert werden. Aufgrund der begrenzten thermischen Stabilität des Katalysators kann jedoch nur ein

\section{Als Katalysatoren bzw. Katalysator- träger können Schwämme insbe- sondere dann Vor- teile gegenüber her- kömmlichen Fest- betten bieten, wenn hohe Fluiddurch- sätze gefordert sind, der Transport der Reaktanden aus der Gasphase zur Katalysatorober- fläche die Reak- tionskinetik hemmt und/oder Reak- tionen eine starke Wärmetönung aufweisen.}

\section{An katalytischen Schwämmen ist in einem weiten Luftzahlbereich eine stabile und schadstoffarme Ver- brennung möglich.}




\section{Ob sich katalytische Schwämme in der Verbrennungstech- nik etablieren wer- den, hängt generell davon ab, ob sich ihre thermische Sta- bilität im Langzeit- betrieb verbessern lässt oder nicht.}

Die tortuose Struktur, die hohe Porosität und die große volumenspezifische Oberfläche sind geeignete Vorraussetzungen für die Anwendung keramischer Schwämme zur Entfernung von Rußpartikeln aus Dieselabgasen.
Teil des Brennstoffs katalytisch verbrannt werden [51].

$\mathrm{Ob}$ sich katalytische Schwämme in der Verbrennungstechnik etablieren werden, hängt generell davon ab, ob sich ihre thermische Stabilität im Langzeitbetrieb verbessern lässt oder nicht.

\subsection{Abgaskatalyse}

Schon vor über 40 Jahren wurde der Einsatz von keramischen Schwämmen für die katalytische Entfernung von Kohlenwasserstoffen aus Automobilabgasen vorgeschlagen [55]. Keramische Wabenkörper-Katalysatoren sind jedoch kostengünstiger herzustellen und weisen eine höhere Stabilität gegenüber mechanischen Schocks und Vibrationen auf. Vermutlich gibt es ein höheres Potenzial für stationäre Anwendungen, beispielsweise im Bereich der katalytischen Entfernung von organischen Komponenten [56, 57] und $\mathrm{NO}_{\mathrm{x}}[58]$ aus $\mathrm{Ab}$ gasströmen.

Ein multifunktionales Reaktorkonzept zur simultanen katalytischen $\mathrm{NO}_{\mathrm{x}}$ - und VOC-Entfernung in Kombination mit der Abtrennung von Partikeln wird in $[29,30]$ vorgestellt. Dabei ist ein keramischer Schwamm, der mit zwei Katalysatoren $\left(\mathrm{V}_{2} \mathrm{O}_{5} \cdot \mathrm{WO}_{3} \cdot \mathrm{TiO}_{2}\right.$ und $\mathrm{MnO}_{\mathrm{x}} \cdot \mathrm{CeO}_{2}$ ) beschichtet wurde, im Inneren eines Gewebefilters für die Partikelabtrennung platziert. Bei dieser Anwendung ist nicht nur ein geringer Druckverlust gefordert, sondern insbesondere die Möglichkeit der allseitigen, d.h. auch radialen, Gasdurchlässigkeit, die beim Wabenkörper nicht gegeben ist. Allerdings besteht hier die Schwierigkeit darin, einen Katalysator zu entwickeln, der bei Temperaturen unter $210{ }^{\circ} \mathrm{C}$, vorgegeben durch die thermische Stabilität des Filtergewebes, ausreichend aktiv ist.

\subsection{Katalytische Rußfilter}

Die tortuose Struktur, die hohe Porosität und die große volumenspezifische Oberfläche sind geeignete Vorraussetzungen für die Anwendung keramischer Schwämme zur Entfernung von Rußpartikeln aus Dieselabgasen. Neben der Filterwirkung ermöglicht die Beschichtung der Schwämme mit geeigneten Katalysatoren einen effektiven Abbrand der Partikel und somit eine regelmäßige Regeneration des Filters.

Frühe Untersuchungen an CordieritSchwämmen $(\sim 10$ PPI, $\varepsilon \sim 0,8)$, beschichtet mit $\mathrm{Fe} / \mathrm{Co}-\mathrm{La}_{2} \mathrm{O}_{3}$-Pt-Katalysatoren, zeigten bereits eine Verbesserung von Filtration und katalytischer Verbrennung der Partikel im Ver- gleich zu axial durchströmten Wabenkörpern und Formkörperschüttungen. Problematisch war jedoch der zu geringe Kontakt zwischen Rußpartikeln und Katalysatorschicht, so dass eine Verbrennung nur oberhalb von $560{ }^{\circ} \mathrm{C} \mathrm{zu}$ beobachten war [59].

Aufgrund der verschärften europaweiten Gesetze über Schadstoffemissionen wurde in den letzten fünf bis sieben Jahren die Forschungstätigkeit auf diesem Gebiet stark intensiviert, was z. B. in [60] zusammengefasst ist. Im Gegensatz zur Filtration durch die Zellwände der Wabenkörpermonolithe (Oberflächenfiltration, „wall flow filter“) funktionieren keramische Schwämme als Tiefenfilter [34]. In aktuellen Untersuchungen wurden keramische Schwämme (50 - 65 PPI, $\varepsilon=0,8-0,9)$ mit Katalysatoren vom Typ $\mathrm{Cs}_{4} \mathrm{~V}_{2} \mathrm{O}_{7}[34,35]$, $\mathrm{CsVO}_{3} \cdot \mathrm{MoO}_{3}$ [31] $\mathrm{Cs}_{2}\left(\mathrm{SO}_{4}\right)_{2} \cdot \mathrm{V}_{2} \mathrm{O}_{5}$ [32] und $\mathrm{Cu} / \mathrm{V} / \mathrm{K} / \mathrm{Cl}[36,37]$ beschichtet, die unter Betriebsbedingungen als Schmelze vorliegen. Dadurch konnte der Kontakt zwischen Rußpartikeln und Katalysatoren verbessert werden. Dies in Kombination mit einer verbesserten Aktivität der Katalysatoren ermöglicht die Verbrennung der Partikel bereits bei Temperaturen unterhalb von $350{ }^{\circ} \mathrm{C}$. Die Filtrationseffizienz für submikrone Partikel liegt bei Schwämmen allerdings nur bei $70-85 \%$.

\subsection{Partielle Oxidation von Kohlen- wasserstoffen zu Synthesegas}

Die partielle Oxidation von Methan [38, 39, $61,62]$, aber auch von höheren Kohlenwasserstoffen [63, 64] zu Synthesegas wird meist bei Temperaturen über $900{ }^{\circ} \mathrm{C}$ und Raumgeschwindigkeiten von mehr als $500000 \mathrm{~L}_{\mathrm{N}} /(\mathrm{L} \mathrm{h})$ an Pt- oder Pt-Rh-Katalysatoren durchgeführt. Diese Bedingungen führen $\mathrm{zu}$ Strömungsgeschwindigkeiten von über $1 \mathrm{~m} / \mathrm{s}$ und Kontaktzeiten von wenigen Millisekunden. Der geringe Druckverlust, die hohe thermische Stabilität und der intensive Austausch von Stoff und Wärme über den gesamten Reaktorquerschnitt machen hier den Einsatz von keramischen Schwämmen als Katalysatorträger interessant.

In [38] werden katalytisch beschichtete keramische Schwämme $\left(a-\mathrm{Al}_{2} \mathrm{O}_{3}, 50\right.$ PPI, $12-20 \%$ $\mathrm{m} / \mathrm{m}$ Pt bzw. Pt-Rh) mit Wabenkörpermonolithen (Cordierit, 400 cpsi, 12 - $14 \%$ m/m Pt) und Netzkatalysatoren (Pt, Pt/Rh) bei der partiellen Oxidation von Methan verglichen. Bei Methanumsätzen von mehr als $80 \%$ werden maximale Selektivitäten $\mathrm{zu} \mathrm{H}_{2}$ von $~ 55 \%$ und zu CO von $\sim 90 \%$ erreicht. Ein signifikanter Einfluss der Katalysatorstruktur auf Umsatz und Selektivitäten konnte jedoch nicht beob- 
achtet werden. Allerdings wurden im Wabenkörper die stärksten Temperaturgradienten gemessen, was für einen besseren Abtransport der Reaktionswärme in Schwämmen und Edelmetallnetzen spricht.

Weitere Studien zeigen, dass bei katalytischen Schwämmen das Ergebnis durch die Porendichte und die Oberflächenbeschaffenheit beeinflusst wird [39]. So wurden an Rhimprägnierten $a$ - $\mathrm{Al}_{2} \mathrm{O}_{3}$-Schwämmen $(20-80$ PPI, $\varepsilon \sim 0,8)$ die höchsten Selektivitäten $\mathrm{zu} \mathrm{H}_{2}$ bzw. CO von jeweils 93 - $95 \%$ bei Umsätzen von $>80 \%$ mit der höchsten PPI-Zahl bzw. den kleinsten Porendurchmessern erreicht. Eine weitere Verbesserung gelang durch das Aufbringen eines „Washcoats“, auf den Rh imprägniert wurde, und durch den Einsatz von $\mathrm{ZrO}_{2}$ dotierten $\mathrm{Al}_{2} \mathrm{O}_{3}$-Schwämmen. Da die katalytische Aktivität der Schwämme ohne Edelmetall $\mathrm{zu}$ vernachlässigen war, wurden diese Effekte mit einer Beschleunigung des Stofftransports zwischen Gasphase und Katalysator erklärt, der unter den vorherrschenden Bedingungen die Kinetik der Reaktion stark beeinflusst: Geringere Porendurchmesser resultieren in kürzeren Diffusionswegen, „Washcoating" und $\mathrm{ZrO}_{2}$-Dotierung in verstärkter Oberflächenrauigkeit.

In einer vergleichenden Studie zur partiellen Oxidation von Propan in einem Mikrostrukturreaktor aus einer FeCr-Legierung und in einem Festbettreaktor mit einer Rh-imprägnierten Schwammpackung wurden am Schwamm höhere Umsätze und $\mathrm{H}_{2}$-Selektivitäten (> $80 \%$ ) bei tieferen Temperaturen erreicht. Als möglicher Grund wird eine bessere Verknüpfung zwischen homogenen und heterogenen Reaktionen aufgeführt. Allerdings bilden sich im keramischen Schwamm wegen des schlechteren Wärmetransports erwartungsgemäß steilere Temperaturgradienten aus als im metallischen Mikrostrukturreaktor, was unter bestimmten Bedingungen zur verstärkten Katalysatordesaktivierung führen kann [64].

\subsection{Ammoniakoxidation und Ammoxidation von Methan}

Über die Oxidation von Ammoniak $\mathrm{zu} \mathrm{NO}_{\mathrm{x}}$ und der Ammoxidation von Methan zu HCN gibt es interessante Arbeiten, in denen evaluiert wird, ob Wabenkörper (Cordierit, 400 2200 cpsi) und keramische Schwämme ( $a$ $\left.\mathrm{Al}_{2} \mathrm{O}_{3}, 30-50 \mathrm{PPI}\right)$, imprägniert mit Pt oder Pt-Rh $(2-20 \% \mathrm{~m} / \mathrm{m})$ eine kostengünstige, langzeitstabilere und selektivere Alternative zu den herkömmlich eingesetzten Pt-Rh-Netzkatalysatoren darstellen [65, 66].

\begin{tabular}{|c|c|c|c|c|}
\hline $\begin{array}{c}\text { Umsatz bzw. } \\
\text { Ausbeute }\end{array}$ & Pt-Rh-Netze ${ }^{1) 2}$ & $\begin{array}{c}\text { Pt-Schwämme } \\
30 \text { PPI, } \\
a-\mathrm{Al}_{2} \mathrm{O}_{3}{ }^{1)}\end{array}$ & $\begin{array}{c}\text { Pt-Schwämme } \\
45 \mathrm{PPI}, \\
a-\mathrm{Al}_{2} \mathrm{O}_{3}{ }^{2}\end{array}$ & $\begin{array}{c}\text { Pt-Wabenkörper } \\
400 \text { cpsi, } \\
\text { Cordierit }^{1)}\end{array}$ \\
\hline $\mathrm{X}\left(\mathrm{CH}_{4}\right)$ & $\sim 0,9$ & 0,93 & 0,97 & 0,89 \\
\hline $\mathrm{X}\left(\mathrm{NH}_{3}\right)$ & $0,83-0,85$ & 0,71 & 0,8 & 0,62 \\
\hline $\mathrm{Y}\left(\mathrm{HCN} / \mathrm{CH}_{4}\right)$ & $0,56-0,63$ & 0,33 & 0,62 & 0,03 \\
\hline $\mathrm{Y}\left(\mathrm{HCN} / \mathrm{NH}_{3}\right)$ & 0,64 & 0,41 & 0,64 & 0,03 \\
\hline
\end{tabular}

1) $\mathrm{CH}_{4} / \mathrm{Luft}=0,18, \mathrm{NH}_{3} / \mathrm{Luft}=0,16[65]$

2) $\mathrm{CH}_{4} / \mathrm{NH}_{3}=1,\left(\mathrm{CH}_{4}+\mathrm{NH}_{3}\right) / \mathrm{O}_{2}=1,8[66]$

Tabelle 4. Vergleich von verschiedenen Katalysatorstrukturen zur Ammoxidation von Methan zu HCN, Werte aus $[65,66]$.

Tab. 4 zeigt einen Auszug aus den Ergebnissen der Untersuchung zur Ammoxidation von Methan. Im Fall von Pt-imprägnierten Schwämmen mit höheren PPI-Zahlen liegen die Umsätze und Ausbeuten im gleichen Bereich wie bei kommerziellen Pt-Rh-Netzkatalysatoren. Da die Notwendigkeit von Rh-Zusätzen entfällt und für eine Beschichtung weit geringere Mengen an Platin benötigt werden, könnten Schwämme tatsächlich eine kostengünstige Packung für Reaktoren in der HCNSynthese darstellen. Die höheren Umsätze und Selektivitäten im Vergleich zu Wabenkörpern und mit zunehmender PPI-Zahl wurden auf einen verbesserten Stoff- und Wärmetransport sowie auf eine verminderte Rückvermischung in der Schwammstruktur zurückgeführt.

\subsection{Oxidative Dehydrierung von Paraffinen zu Olefinen}

$\mathrm{Zu}$ den Hochtemperaturprozessen mit kurzen Kontaktzeiten, bei denen katalytische Schwämme bereits erprobt wurden, zählt auch die Oxydehydrierung von Alkanen zu Olefinen [39 - 44]. In den bisherigen Untersuchungen stand die Umsetzung von Ethan zu Ethen im Mittelpunkt. An Pt-imprägnierten Schwämmen $\left(a-\mathrm{Al}_{2} \mathrm{O}_{3}, 80\right.$ PPI, $\left.2,3 \% \mathrm{Pt} \mathrm{m} / \mathrm{m}\right)$ werden bei ca. $900{ }^{\circ} \mathrm{C}$ Ethanumsätze von $>80 \%$ und Selektivitäten zu Ethen von etwa $70 \%$ erreicht [40].

Durch eine Dotierung der Pt-Schicht mit Sn und die Zugabe von $\mathrm{H}_{2}$ im Reaktorzulauf kann die Ethenselektivität sogar auf über 80 \% gesteigert werden, was die Basis für ein konkurrenzfähiges Verfahren zum "Steamcracking“ schaffen könnte [43]. Im Gegensatz zur partiellen Oxidation von Alkanen zu Synthesegas wurde beobachtet, dass Schwammmaterial und Porendichte nur einen geringen Einfluss auf Ethanumsätze und Ethenselektivitäten haben. Wurde jedoch Pt auf einen mit $\gamma-\mathrm{Al}_{2} \mathrm{O}_{3}$ "washcoat“ beschichteten Schwamm imprä-
Da die Notwendigkeit von Rh-Zusätzen entfällt und für eine Beschichtung weit geringere Mengen an Platin benötigt werden, könnten Schwämme tatsächlich eine kostengünstige Pakkung für Reaktoren in der HCN-Synthese darstellen. 
gniert, verringerten sich Aktivität und Selektivität. Als mögliche Ursachen dafür wurden eine Blindaktivität des $\gamma-\mathrm{Al}_{2} \mathrm{O}_{3}$ in der Umsetzung von Ethen und eine Stofftransporthemmung im porösen „washcoat" angenommen [39].

Eine kürzlich erschienene Studie vergleicht die Wirkung von $a-\mathrm{Al}_{2} \mathrm{O}_{3}$-Schwämmen unterschiedlicher PPI-Zahl $(45-80$ PPI, $\varepsilon \sim 0,83)$ mit Wabenkörpern (Cordierit, 200 - 1200 cpsi) [44]. Diese Packungen wurden entweder mit Platin oder mit $\mathrm{LaMnO}_{3}$-Perowskit beschichtet. Neben einer höheren Aktivität und Selektivität der Perowskite im Vergleich zu Platin wurde im Gegensatz zu [39] eine für Umsätze und Selektivitäten optimale Poren- bzw. Zelldichte für beide Träger gefunden. Erklärt wird dieses Optimum mit einem besonders günstigen Verhältnis von Leeraumanteil zu Oberfläche, bei dem eine Kopplung von homogenen und heterogenen Reaktionen am besten gelingt (s. a. Abschnitt 5.3). Diese Annahme wird für die Wabenkörper durch Modellrechnungen gestützt. Höhere Umsätze und Selektivitäten an den Schwämmen werden wiederum durch den verbesserten Stoff- und Wärmetransport begründet. Zudem zeigen abschätzende Rechnungen eine $\mathrm{zu}$ vernachlässigende Rückvermischung in Schwämmen, während diese in Wabenkörpern trotz höherer Reynolds-Zahlen $\mathrm{zu}$ berücksichtigen ist.

\subsection{Partielle Oxidation von Methanol zu Formaldehyd}

Keramische Schwammpackungen könnten aufgrund ihrer offenzelligen Struktur und dem geringen Druckverlust auch im Silberkontaktverfahren für die Formaldehydsynthese eingesetzt werden. Erste Studien zeigen, dass an Schwamm-geträgertem Silber bei Temperaturen von $650{ }^{\circ} \mathrm{C}$ höhere Ausbeuten an Formaldehyd erzielt werden als mit $a-\mathrm{Al}_{2} \mathrm{O}_{3}$-geträgertem Silber. Die Langzeitstabilität ist allerdings bei geträgerten Systemen durchweg schlechter als bei den kommerziell eingesetzten Silbervollkristallen [67]. Dies ist auf die Migration und Aggregation des Silbers auf der Trägeroberfläche zurückzuführen, was eventuell durch eine Dotierung mit Zr- und Ce-Ionen verbessert werden kann [68].

\subsection{Partielle Oxidation von o-Xylol}

Im Gegensatz zu den vorher genannten Oxidationen, die in technischen Prozessen adiabatisch durchgeführt werden, ist die partielle Oxidation von o-Xylol zu Phthalsäureanhydrid
(PSA) ein stark exothermer Prozess, der bei etwa $360-400{ }^{\circ} \mathrm{C}$ möglichst isotherm geführt werden muss. Übertemperaturen führen zum Verlust der PSA-Selektiviität und zur Desaktivierung der promotierten $\mathrm{V}_{2} \mathrm{O}_{5} / \mathrm{TiO}_{2}$-Katalysatoren. Diese werden in industriellen Reaktoren als Schüttung von Schalenkatalysatoren eingesetzt, um eine Beeinträchtigung von Aktivität und Selektivität durch Stofftransporthemmungen im Katalysator zu unterbinden.

In Simulationsstudien wurde nun gezeigt, dass die guten Wärmeübertragungseigenschaften, die hohe Oberfläche und der geringe Druckverlust von Schwammpackungen neue Möglichkeiten für die Betriebsweise von PSAReaktoren zulassen [69, 70]. Geringere Temperaturgradienten in den schwammgepackten Reaktoren ermöglichen höhere o-Xylol-Eingangskonzentrationen und Reaktortemperaturen, aber auch den Einsatz von größeren Katalysatormengen oder aktiveren Katalysatoren bei höheren Gasdurchsätzen. Jede diese Veränderungen oder eine Kombination aus diesen führt zu einer deutlichen Erhöhung der PSARaum-Zeit-Ausbeuten bei höheren PSA-Selektivitäten. Zusätzlich besteht wegen der guten Wärmeabfuhr die wirtschaftlich attraktive Option, den Durchmesser der Rohre im Rohrbündelreaktor zu vergrößern. Die experimentelle Validierung der Modellrechnungen steht noch aus, ist aber in Arbeit.

Bei den bereits durchgeführten experimentellen Untersuchungen standen noch die katalytische Beschichtung der Schwämme im Mittelpunkt sowie der Nachweis, dass die rein katalytischen Eigenschaften der Schwammpackungen in der PSA-Synthese mit jenen von konventionellen Kontakten mithalten können. Tatsächlich zeigen die Ergebnisse, dass keramische Schwämme (20 PPI, $\varepsilon=0,85$, Mullit), direkt beschichtet mit $\mathrm{V}_{2} \mathrm{O}_{5} / \mathrm{TiO}_{2}$-Nanopartikeln, die durch Flammensprühpyrolyse hergestellt wurden, extrem aktiv sind und eine etwa 10-fache PSA-Produktivität (Massenstrom PSA/Masse Katalysator) ergeben [48].

\subsection{Reformierung von Kohlenwasser- stoffen zu Synthesegas}

Die Reformierung von Kohlenwasserstoffen zur Erzeugung von Synthesegas ist ein stark endothermer Prozess. Bei Temperaturen um $800{ }^{\circ} \mathrm{C}$ muss eine effiziente Wärmeeinkopplung in die Reaktoren gewährleistet werden, da es sonst zur Ausbildung von Temperaturgradienten bzw. „Cold Spots“ kommt, die eine Erniedrigung der Reaktionsgeschwindigkeit zur Folge haben. Auch hier zeigen Modellrechnungen, dass der bessere Wärmeeintrag und 
der geringere Druckverlust in Schwämmen eine Kosten sparende Rekonfiguration der Reformer ermöglichen sollten [2]. Experimentelle Daten dazu wurden noch nicht veröffentlicht.

Untersuchungen zur Reformierung von Methan mit $\mathrm{CO}_{2}$ zeigten, dass an Rh-imprägnierten $a$ - $\mathrm{Al}_{2} \mathrm{O}_{3}$-Schwämmen (20 PPI, $\varepsilon=0,85$ ) eine um den Faktor 10 höhere Reaktionsgeschwindigkeit erreicht werden kann als mit Schüttungen aus kommerziellen $\mathrm{Rh} / a-\mathrm{Al}_{2} \mathrm{O}_{3}$ Pellets. Als Grund dafür wird eine Hemmung der Kinetik durch Porendiffusion in den Pellets angegeben, die in der dünnen Katalysatorschicht auf den Schwämmen nicht oder nur stark vermindert auftritt. Die Messungen deuten auch eine verbesserte Einkopplung von Wärme, insbesondere über Strahlung, in den Reaktor an [71].

Seit längerem wird bereits der Betrieb von Reaktoren für die Methanreformierung untersucht, die mit Solarenergie betrieben werden sollen. Darin werden keramische Schwämme als Absorber für die Solarenergie und als Träger für verschiedene Katalysatoren eingesetzt. Im Vergleich zu anderen Strukturen gewährleisten keramische Schwämme eine effiziente und gleichmäßige Absorption der Strahlung und eine homogene Temperaturverteilung bei ausreichender thermischen Stabilität [72 - 76].

\subsection{MTO (Methanol to Olefins)}

In [77] wurde die Umsetzung von Methanol an mit H-ZSM-5 beschichteten Schwämmen (45 PPI) untersucht und mit einer Schüttung aus zylindrischen Extrudaten (H-ZSM-5 gebunden mit $\gamma-\mathrm{Al}_{2} \mathrm{O}_{3}$ ) verglichen. Bei den Schwämmen wurde die Zeolith-Schichtdicke variiert. Die Experimente haben erwiesen, dass die Olefinselektivität an Schwammpackungen deutlich höher ist als bei den Extrudaten und mit abnehmender Schichtdicke zunimmt. Auch ein höheres Propen/Ethen-Verhältnis wurde bei Schwammpackungen erreicht. Diese Ergebnisse lassen sich durch verminderte Stofftransporthemmungen in der dünnen Schicht auf der Schwammpackung erklären. Obwohl die Schwämme eine geringere Menge an Zeolith pro Packungsvolumen enthalten als die Extrudate, sind die maximal erreichbaren OlefinRaum-Zeit-Ausbeuten pro Reaktorvolumen dennoch um einen Faktor von bis zu 2,5 höher.

\section{Zusammenfassung}

Feste Schwämme aus unterschiedlichen keramischen Werkstoffen sind für den Einsatz als strukturierte Packungen in vielen reaktionstechnischen Anwendungen interessant. Hinsichtlich Druckverlust, Durchmischung, Stoffund Wärmetransport vereinigen sie offensichtlich Vorzüge von Wabenkörpern und von Festbettschüttungen. Diese Bewertung hat jedoch derzeit noch einen mehr oder weniger qualitativen Charakter, denn es gibt noch einen großen Forschungsbedarf im Hinblick auf die quantitative Beschreibung von Fluiddynamik, Wärme- und Stofftransport in Schwämmen auf Basis eines realitätsnahen Modells für ihre Struktur.

Die derzeit verfügbaren Untersuchungen auf dem Gebiet der katalytischen Reaktionstechnik zeigen schon eine große Vielfalt an möglichen Anwendungen auf und deuten auf potenzielle Vorteile gegenüber herkömmlichen Festbetten hin. Eine robuste Modellierung von Reaktoren, die mit katalytischen Schwammpackungen beaufschlagt sind, ist aber aus den oben genannten Gründen noch nicht möglich. Insofern stehen belastbare quantitative Kriterien für den zweckmäßigen Einsatz katalytischer Schwammpackungen noch nicht zur Verfügung. Daher ist es noch nicht möglich, durch Vorberechnungen jene strukturellen Merkmale zu identifizieren, die ein optimaler Schwamm für eine gewünschte Anwendung aufweisen müsste.

Eine Forschergruppe der Deutschen Forschungsgemeinschaft (FOR 583) an der Universität Karlsruhe hat es sich zum Ziel gesetzt, hier Fortschritte zu erzielen, indem sie Grundlagenforschung an den Material- und Transporteigenschaften keramischer Schwämme mit der Untersuchung verschiedener verfahrenstechnischer Anwendungen verbindet.

Die Autoren danken der DFG für die Förderung im Rahmen der Forschergruppe 583 „Feste Schwämme - Anwendung monolithischer Netzstrukturen in der Verfahrenstechnik“. F. C. Patcas dankt dem Land Baden-Württemberg für die finanzielle Unterstützung im Rahmen des Margarete-von-WrangellHabilitationsstipendiums.

Eingegangen am 2. März 2006

\section{Formelzeichen}

$\begin{array}{lll}c_{\mathrm{f}} & {\left[\mathrm{J} \mathrm{kg}^{-1} \mathrm{~K}^{-1}\right]} & \begin{array}{l}\text { Wärmekapazität des } \\ \text { Fluids }\end{array} \\ & & \text { Zelldurchmesser } \\ d_{\mathrm{C}} & {[\mathrm{m}]} & \text { Fensterdurchmesser } \\ d_{\mathrm{F}} & {[\mathrm{m}]} & \end{array}$
Feste Schwämme aus unterschied- lichen keramischen Werkstoffen sind für den Einsatz als strukturierte $\mathrm{Pa}$ - ckungen in vielen reaktionstechni- schen Anwendun- gen interessant. 


\begin{tabular}{|c|c|c|}
\hline$D_{\mathrm{m}}$ & {$\left[\mathrm{m} \mathrm{s}^{-2}\right]$} & $\begin{array}{l}\text { molekularer Diffusions- } \\
\text { koeffizient }\end{array}$ \\
\hline$d_{\mathrm{P}}$ & {$[\mathrm{m}]$} & Porendurchmesser \\
\hline$d_{\mathrm{R}}$ & {$[\mathrm{m}]$} & Rohrdurchmesser \\
\hline$h_{\text {eff }}$ & {$\left[\mathrm{W} \mathrm{m}{ }^{-2} \mathrm{~K}^{-1}\right]$} & $\begin{array}{l}\text { Wärmedurchgangs- } \\
\text { koeffizient }\end{array}$ \\
\hline$h$ & {$\left[\mathrm{~W} \mathrm{~m}{ }^{-2} \mathrm{~K}^{-1}\right]$} & $\begin{array}{l}\text { Wärmeübergang- } \\
\text { koeffizient }\end{array}$ \\
\hline$k_{\mathrm{m}}$ & {$\left[\mathrm{m} \mathrm{s}^{-1}\right]$} & Stoffübergangskoeffizient \\
\hline$S_{\mathrm{V}}$ & {$\left[\mathrm{m}^{-1}\right]$} & $\begin{array}{l}\text { spezifische Oberfläche be- } \\
\text { zogen auf Bettvolumen }\end{array}$ \\
\hline$l$ & {$[\mathrm{~m}]$} & Stegdicke \\
\hline 11 & {$\left[\mathrm{~m} \mathrm{~s}^{-1}\right]$} & Leerrohrgeschwindigkeit \\
\hline
\end{tabular}
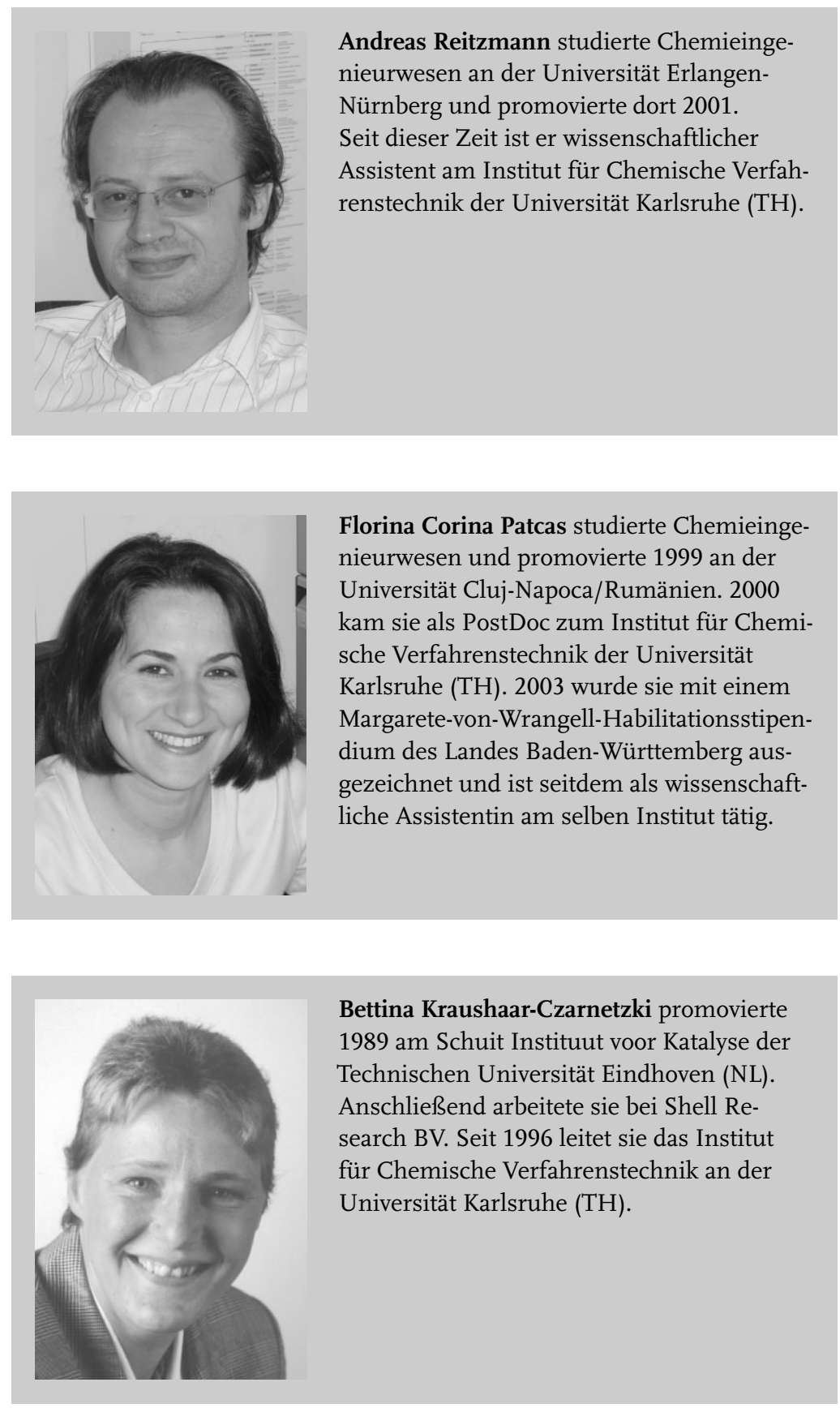

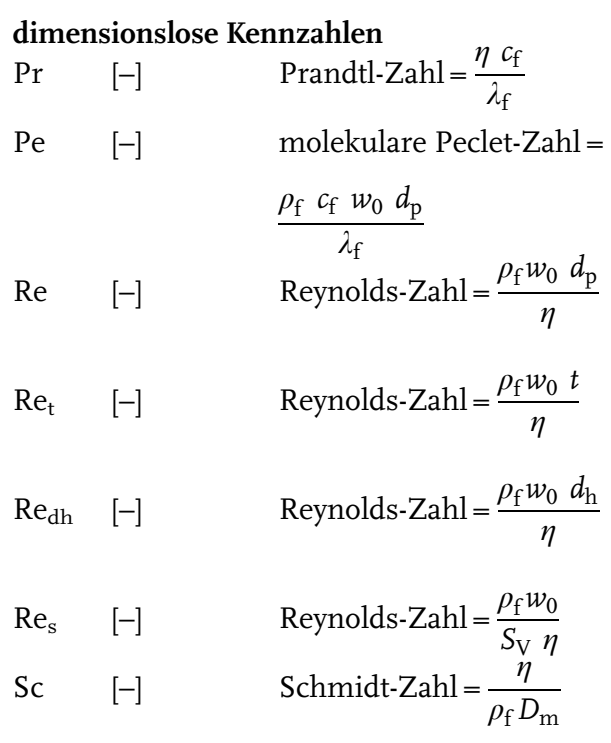

griechische Buchstaben

\begin{tabular}{|c|c|c|}
\hline$\varepsilon$ & {$[-]$} & Leeraumanteil \\
\hline$\eta$ & {$[\mathrm{Pa} \mathrm{s}]$} & $\begin{array}{l}\text { dynamische Viskosität } \\
\text { des Fluids }\end{array}$ \\
\hline$\gamma_{\mathrm{s}}$ & {$\left[\mathrm{W} \mathrm{m} \mathrm{m}^{-1} \mathrm{~K}^{-1}\right]$} & $\begin{array}{l}\text { Wärmeleitfähigkeit } \\
\text { des Feststoffs }\end{array}$ \\
\hline$\gamma_{\mathrm{f}}$ & {$\left[\mathrm{W} \mathrm{m} \mathrm{m}^{-1} \mathrm{~K}^{-1}\right]$} & $\begin{array}{l}\text { Wärmeleitfähigkeit } \\
\text { des Fluids }\end{array}$ \\
\hline$\gamma^{0}$ & {$\left[\mathrm{~W} \mathrm{~m}{ }^{-1} \mathrm{~K}^{-1}\right]$} & Ruhewärmeleitfähigkeit \\
\hline$\gamma_{\text {eff, rad }}$ & {$\left[\mathrm{W} \mathrm{m}{ }^{-1} \mathrm{~K}^{-1}\right]$} & $\begin{array}{l}\text { effektive radiale Wärme- } \\
\text { leitfähigkeit }\end{array}$ \\
\hline$\rho$ & {$\left[\mathrm{kg} \mathrm{m}^{-3}\right]$} & Schwammpackungsdichte \\
\hline$\rho_{\mathrm{S}}$ & {$\left[\mathrm{kg} \mathrm{m}^{-3}\right]$} & Stegdichte \\
\hline$\rho_{\mathrm{f}}$ & {$\left[\mathrm{kg} \mathrm{m}^{-3}\right]$} & Fluiddichte \\
\hline
\end{tabular}

A. Reitzmann

(andreas.reitzmann@ciw.uni-karlsruhe.de),

F. C. Patcas,

\section{B. Kraushaar-Czarnetzki,}

Universität Karlsruhe (TH), Institut für

Chemische Verfahrenstechnik, Kaiserstraße 12,

D-76185 Karlsruhe, Germany.

\section{Literatur}

[1] http://www.foseco.de/;

http://www.vesuvius.com/;

http://www.techceramics.com/pdf/

Reticel_Bro.pdf

[2] http://www.azom.com/details.asp? ArticleID=911; http://www.azom.com/ details.asp?ArticleID=1869;

[3] J. Adler, G. Standke, Keram. Z. 2003, 55 (10), 786.

[4] M. V. Twigg, J. T. Richardson, Stud. Surf. Sci. Catal. 1995, 91, 345.

[5] F. Durst, K. Pickenäcker, D. Trimis, Gas-Erdgas 1997, 138 (2), 116.

[6] M. V. Twigg, J. T. Richardson, Trans. Inst. Chem. Eng. 2002, 80, 183. 
[7] L. J. Gibson, M. F. Ashby, in Cellular Solids: Structure and Properties, Pergamon Press, Oxford 1988, 23.

[8] D. Weaire, S. Hutzler, in The Physics of Foams, Oxford University Press, Oxford 1999, 159.

[9] http://www.susqu.edu/facstaff/b/brakke/ kelvin/kelvin.html

[10] F. C. Buciuman, B. Kraushaar-Czarnetzki, Ind. Eng. Chem. Res. 2003, 42, 1863.

[11] J. Adler, G. Strandke, Keram. Z. 2003, 55 (9), 694.

[12] J. T. Richardson, Y. Peng, D. Remue, Appl. Catal. A: General 2000, 204, 19.

[13] R. Rice, in Cellular Ceramics (Eds: M. Scheffler, P. Colombo), Wiley-VCH, Weinheim 2005, 291

[14] M. D. M. Innocentini, P. Sepulveda, F. S. Ortega, in Cellular Ceramics (Eds: M. Scheffler, P. Colombo), Wiley-VCH, Weinheim 2005, 313.

[15] E. A. Moreira, M. D. M. Innocentini, J. R. Coury, J. Eur. Ceram. Soc. 2004, 24, 3209.

[16] P. Du Plessis, A. Montillet, J. Comiti, J. Legrand, Chem. Eng. Sci. 1994, 49, 3545.

[17] J. T. Richardson, D. Remue, J.-K. Hung, Appl. Catal. A: General 2003, 250, 319.

[18] L. Giani, G. Gropi, E. Tronconi, Ind. Eng. Chem. Res. 2005, 44, 4993.

[19] A. Schlegel, P. Benz, S. Buser, Wärme- und Stoffübertragung 1993, 28, 259.

[20] L. Giani, G. Groppi, E. Tronconi, Ind. Eng. Chem. Res. 2005, 44, 9078.

[21] L. Younis, R. Viskanta, Int. J. Heat Mass Transfer 1993, 36 (6), 1425.

[22] X. Fu, R. Viskanta, J. P. Gore, Int. Comm. Heat Mass Transfer 1998, 25 (2), 151.

[23] A. Bhattacharya, V. V. Calmidi, R. I. Mahajan, Int. J. Heat Mass Transfer 2002, 45, 1017.

[24] Y. Peng, J. T. Richardson, Appl. Catal. A: General 2004, 266, 235.

[25] S. Decker et al., Thermisch beaufschlagte Porenkörper und deren Durchströmungs- und Wärmeübertragungseigenschaften, Abschlussbericht DFG-Projekt DU 101/55-1, Bonn 2002.

[26] W. Schabel, M. Abu-Khader, B. Dietrich, H. Martin, in Proc. 13th Int. Heat Transfer Conf. (IHTC-13), Sydney, Australia, 2006, akzeptiert.

[27] T. Boger, A. K. Heibel, C. M. Sorensen, Ind. Eng. Chem. Res. 2004, 43 (6), 4602.

[28] L. Giani, C. Cristiani, G. Groppi, E. Tronconi, Appl. Catal. B: Environ. 2006, 62, 121.

[29] D. Fino et al., Chem. Eng. Sci. 2004, 59, 5329.

[30] D. Fino et al., Ind. Eng. Chem. Res. 2005, 44, 9542.

[31] B. A. A. L. van Stetten et al., Catal. Today 1999, 53, 613.

[32] B. A. A. L. van Stetten et al., Topics Catal. 2001, 16/17 (1-4), 275.

[33] F. C. Buciuman, B. Kraushaar-Czarnetzki, Catal. Today 2001, 69, 337.

[34] G. Saracco et al., Catal. Today 2000, 60, 33.

[35] D. Fino, G. Saracco, V. Speccia, Chem. Eng. Sci. 2002, 57, 4955.

[36] P. Russo et al., Topics Catal. 2003, 22 (1/2), 123.

[37] P. Ciambelli et al., Chem. Eng. Sci. 2005, 60, 1619.
[38] D. A. Hickman, L. D. Schmidt, J. Catal. 1992, 138, 267.

[39] A. S. Bodke, S. S. Bharadway, L. D. Schmidt, J. Catal. 1998, 179, 138.

[40] M. Huff, L. D. Schmidt, J. Phys. Chem. 1993, 97, 11815.

[41] M. Huff, L. D. Schmidt, J. Catal. 1994, 149, 127.

[42] M. Huff, L. D. Schmidt, J. Catal. 1995, 155, 82.

[43] A. S. Bodke et al., Science 1999, 285, 712.

[44] F. Donsi et al., Catal. Today 2005, 105, 551.

[45] G. B. F. Seijger et al., Micropor. Mesopor. Mater. 2000, 39, 195

[46] F. Scheffler et al., Adv. Appl. Ceram. 2005, 104 (1), 1.

[47] B. Schimmöller, H. Schulz, S. E. Pratsinis, A. Bareiss, A. Reitzmann, US Patentanmeldung 60/694954, eingereicht: 29.06.2005.

[48] B. Schimmöller, et al., J. Catal. 2006, im Druck. DOI: 10.1016/j.jcat.2006.02.027

[49] M. Haruta, Y. Suoma, H. Sano, J. Hydrogen Energy 1982, 7, 729.

[50] T. Inui, T. Kuroda, T.Otowa, J. Fuel Soc. Jpn. 1985, 64, 270.

[51] A.Schlegel, S. Buser, P. Benz, H. Bockhorn, F. Mauss, in 25th Symposium (International) on Combustion, The Combustion Institute, Pittsburgh 1994, 1019.

[52] C. T. Goralski, L. D. Schmidt, Catal. Lett. 1996, $42,47$.

[53] I. Cerri, G. Saracco, V. Specchia, Catal. Today 2000, 60, 21.

[54] K. Mangold, G. Foerster, W. Taetaner, DE Offenlegungsschrift 3732653, 1989.

[55] K. Schwartzwalder, A. Somers, US Patent 3090094, 1963.

[56] C. T. Goralski, L. D. Schmidt, W. L. Brown, AIChE J. 1998, 44 (8), 1880.

[57] A. N. Pestryakov, V. V. Lunin, N. E. Bogdanchikova, in Catalysis in Applications (Ed: S. D. Jackson et al.), Royal Society of Chemistry, Cambridge, 2003, 216

[58] G. B. F. Seijger et al., Chem. Eng. Sci. 2001, 56, 849.

[59] T. Inui, T. Otowa, Appl. Catal. 1985, 14, 83

[60] B. A. A. L. van Stetten, M. Makee, J. A. Moulijn, Catal. Rev. - Sci. Eng. 2001, 43, 489.

[61] K. A. Vonkeman, L. L. G. Jacobs (Shell International Research), EP Patent 0576096B1, 1993.

[62] U. Friedle, G. Veser, Chem. Eng. Sci. 1999, 54, 1325.

[63] J. J. Krummenacher, K. N. West, L. D. Schmidt, J. Catal. 2003, 215, 332.

[64] I. Aartun et al., Catal. Today 2005, 105, 469.

[65] D. A. Hickman, M. Huff, L. D. Schmidt, Ind. Eng. Chem. Res. 1993, 32, 809.

[66] S. S. Bharadwaj, L. D. Schmidt, Ind. Eng. Chem. Res. 1996, 35, 1524.

[67] A. N. Pestryakov et al., Catal. Commun. 2003, 4, 327.

[68] A. N. Pestryakov, N. E. Bogdanchikova, A. KnopGericke, Catal. Today 2004, 91-92, 49.

[69] A. Reitzmann, P. Pias, A. Bareiss, B. KraushaarCzarnetzki, in Proc. DGMK/SCI - Conf. „Oxidation and Functionalization: Classical and Alternative Routes (Eds: S. Ernst, E. Gallei, J. A. Lercher, 
S. Rossini, E. Santacesaria, DGMK 2005-2, Frankfurt 2005, 107.

[70] A. Reitzmann, A. Bareiss, B. Kraushaar-Czarnetzki, Oil Gas Europ. Mag. 2006, im Druck.

[71] J. T. Richardson, M. Garrait, J.-K. Hung, Appl. Catal. A: General 2003, 255, 69.

[72] T. Fend et al., Energy 2004, 29, 823.
[73] R. E. Hogan et al., Chem. Eng. Sci. 1990, 45, 2751.

[74] J. Völter et al., Chem. Ing. Tech. 1997, 69 (1+2), 83.

[75] R. Tamme et al., J. Solar Energy Eng. 2001, 123, 161.

[76] T. Kodama et al., Energy \& Fuels 2003, 17, 914.

[77] F. C. Patcas, J. Catal. 2005, 231, 194. 